\title{
El estudio de las fortalezas del carácter en niños: Relaciones con el bienestar psicológico, la deseabilidad social y la personalidad
}

\author{
Aldana Sol Grinhauz ${ }^{1}$
}

\begin{abstract}
Artículo
Material original autorizado para la publicación en la revista Psicodebate. Facultad de Ciencias Sociales. Universidad de Palermo.

Recibido 28-10-2014 | Aceptado 01-06-2015
\end{abstract}

\section{Resumen}

Este trabajo tuvo como objetivo corroborar la existencia de asociaciones entre las fortalezas del carácter, el bienestar psicológico, la deseabilidad social y la personalidad en niños de 10 a 12 años. Participaron de este estudio 518 niños (254 varones y 263 mujeres) que tenían entre 10 y 12 años y que asistían a escuelas públicas y privadas de la Ciudad de Buenos Aires (Argentina). Se administraron: una encuesta sociodemográfica, una encuesta para evaluar los cinco elementos de la teoría PERMA de acceso al bienestar, el Inventario de Virtudes y Fortalezas para Niños, el Cuestionario de Personalidad Infantil y la Escala de Deseabilidad Social Infantil. Los resultados mostraron que, al igual que en estudios previos, la vitalidad, la esperanza y la curiosidad fueron las fortalezas que más se asociaron con la satisfacción vital. A su vez, se encontraron correlaciones estadísticamente significativas entre los cinco elementos de la teoría PERMA de acceso al bienestar (Seligman, 2011) y la mayoría de las fortalezas del carácter. Por su parte, las fortalezas también se encontraron asociadas al modelo de los Cinco Grandes Factores de la Personalidad y la deseabilidad social. Finalmente, se hallaron diferencias en la presencia de las fortalezas del carácter de acuerdo al sexo y la edad.

Palabras Clave: fortalezas, niños, bienestar, deseabilidad social, personalidad, psicología positiva.

1 Universidad de Buenos Aires - Argentina; solgrin@yahoo.com.ar 


\section{Abstract}

This study aimed to verify the existence of associations between character strengths, psychological welfare, social desirability and personality in children of 10 to 12 years old. 518 children ( 254 boys and 263 girls) between 10 and 12 years old and attending public and private schools in the City of Buenos Aires (Argentina) participated on this study. The following were administered: a socio-demographic survey; a survey to assess the PERMA's access to welfare theory five elements, the Virtues and Strengths Inventory for Children; Children's Personality Questionnaire and Children's Social Desirability Scale. The results showed that, as in previous studies, vitality, hope and curiosity were the strengths most associated with life satisfaction. At the same time, statistically significant correlations between the PERMA's access to welfare theory five elements (Seligman, 2011) and most of the character's strengths were found. Meanwhile, the strengths were also found associated with the Five Great Personality Factors model and social desirability. Finally, differences in the presence of the character strengths according to sex and age were found.

Keywords: strengths, children, welfare, social desirability, personality, positive psychology. 
Según Seligman y Csikszentmihalyi (2000) antes de la Segunda Guerra Mundial, la Psicología tenía tres misiones: curar la enfermedad mental, hacer las vidas de las personas más plenas e identificar y alimentar el talento. Sin embargo, los autores consideran que la única misión que prosperó hasta nuestros días es el estudio y tratamiento de enfermedades mentales, pero se han descuidado las otras dos misiones.

No obstante, a finales del siglo XX, de la mano de Martin Seligman, surge un área de la Psicología Científica decidida a orientar sus investigaciones hacia todo aquello que hace que la vida merezca ser vivida, interesándose en temas tales como la experimentación y promoción de emociones positivas y la identificación y potenciación de rasgos positivos presentes en todos los seres humanos, como por ejemplo: la bondad, la humildad y la gratitud, entre otras (Seligman \& Csikszentmihalyi, 2000). Esta nueva área se denominó Psicología Positiva.

Para abordar este propósito, en el 2002, Seligman publica un libro llamado $L a$ auténtica felicidad (authentic happiness), en el cual plantea que para el desarrollo de una vida plena y feliz hacen falta tres elementos. El primero consiste en incrementar la experimentación de emociones positivas durante la mayor parte del tiempo.

El segundo elemento teoriza que el placer se deriva del compromiso con la tarea efectiva y con la capacidad de experimentar flow. Si la persona es consciente de sus fortalezas personales, al aplicarlas en una tarea concreta, alcanzará un alto grado de compromiso con esa actividad (Seligman, 2002). En esta segunda vía se resalta también la importancia de identificar los rasgos positivos que las personas tienen en su carácter tales como las virtudes y fortalezas. Se sostiene que la práctica de rasgos positivos, tales como la bondad, el amor o la gratitud entre otros, son una fuente abundante de emociones positivas y gratificaciones. Esto representa la clave de la prevención, ya que estas emociones positivas pueden actuar como amortiguadores contra la enfermedad mental (Peterson \& Seligman, 2004). Por otra parte, la práctica frecuente de los rasgos positivos produce efectos favorables, no solo en los contextos familiar, laboral, educativo y comunitario (Peterson \& Park, 2009), sino también en la calidad de las relaciones interpersonales que en dichos contextos se generan (Seligman, 2002).

El tercer elemento tiene que ver con el significado de vida y consiste en la aplicación de las fortalezas personales para ayudar a los demás y hacer que los demás puedan desarrollar sus potencialidades. Este proceso se da a través de las instituciones: la familia, el trabajo, la escuela, la comunidad (Seligman, Parks, \& Steen, 2006; Steger, 2009).

En el 2009, con motivo del Primer Congreso Mundial de Psicología Positiva organizado por la Asociación Internacional de Psicología Positiva (International Positive Psychology Association; IPPA), Seligman propuso sumar a esta conceptualización tripartita una cuarta vía: el establecimiento de relaciones 
sociales positivas. Este autor hizo hincapié en la importancia de llevar una vida en comunidad, en la cual la persona pueda compartir con otros seres las experiencias de su vida, debido a que las buenas relaciones con otras personas -amigos, familiares y compañeros de trabajo- son el factor que contribuye de manera más importante a la buena vida psicológica.

En el 2011, Seligman publica un nuevo libro llamado Flourish: a visionary new understanding of happines and Well Being, traducido como: Florece: una comprensión nueva y visionaria de la felicidad y el bienestar, en el cual revisa el modelo de felicidad propuesto en el 2002. Este autor propone cambiar el término felicidad por el de bienestar (well being) desmarcándose de esa idea aristotélica de que todas las motivaciones humanas quedan reducidas a una sola (la felicidad). El autor aboga, desde una revisión de su propia teoría de la auténtica felicidad, por un concepto más diversificado, el bienestar.

En este libro, Seligman (2011) anuncia la teoría del bienestar bajo el nuevo modelo denominado PERMA, cuyas letras hacen alusión a cada uno de los cinco elementos esenciales para alcanzar el bienestar: las emociones positivas (positive emotions), el compromiso (engagement), las relaciones positivas (positive relations), el significado de la vida (meaning) y suma un nuevo elemento: la autorrealización o autopercepción de logros (accomplishment). Cada uno de estos cinco elementos cumple con tres condiciones: 1 . Contribuyen al bienestar; 2 . Cada quien los persigue por su propio bien, no necesariamente para poder obtener cualquier otro de los cuatro elementos; y 3 . Cada elemento es definido y medido independientemente del resto de los elementos.

Con relación al quinto elemento - la autopercepción de logros- Seligman (2011) lo describe como la tendencia del ser humano a querer prosperar y superar sus límites a través de sus acciones. Esto tiene que ver con la maestría de ciertos talentos, el alcance de metas, el ganar o perder como consecuencia de un esfuerzo determinado (Seligman, 2011).

$\mathrm{Al}$ anunciar la teoría del bienestar, Seligman (2011) modifica el objetivo primordial de la Psicología Positiva; el mismo consiste ahora en promover el florecimiento humano (human flourishing) a partir del incremento de los cinco elementos que componen el bienestar. También, otorga un lugar más jerárquico a los rasgos positivos y más particularmente a las fortalezas del carácter. Esto quiere decir que estas últimas apuntalan a los cinco elementos de la teoría PERMA y no solamente al segundo elemento -el compromiso- El despliegue de las fortalezas del carácter contribuye a una vida con más significado y compromiso, con más emociones positivas, con relaciones sociales más sanas y duraderas y con mayores logros.

Conforme a esto último, la Psicología Positiva ha dedicado especial atención al estudio científico del carácter definiendo a este último como una familia bien 
conformada de rasgos positivos. (Seligman \& Csikszentmihalyi, 2000). Para la Psicología Positiva, volver a estudiar el carácter implica centrarse en la excelencia moral, en aquellos rasgos positivos que permiten una comprensión profunda del buen vivir psicológico.

De acuerdo con lo planteado, Peterson y Seligman (2004) realizaron una clasificación de los rasgos positivos denominada Valores en Acción (Values in Action) con la intención de que fuera la contraparte del Manual Diagnóstico y Estadístico de los Trastornos Mentales. Estos autores consideraron que contar con una clasificación permitiría establecer un vocabulario común acerca de los rasgos positivos que guíe la investigación, ordene y construya instrumentos para evaluarlos, y se desarrollen intervenciones para promoverlos. De esta forma, los autores presentaron el manual Character Strengths and Virtues. A handbook and classification donde identificaron seis virtudes presentes en la mayoría de las tradiciones filosóficas y religiosas de Oriente y Occidente: el coraje, la justicia, la humanidad, la templanza, la sabiduría y la trascendencia. A su vez, cada una de estas virtudes se asociaba con un determinado número de 24 fortalezas de carácter (ver tabla 1.)

Asimismo, estos autores plantearon que el buen carácter está formado por diversos componentes que se encuentran en diferentes niveles de abstracción. Las virtudes representan los componentes más abstractos. En un nivel menor de abstracción se ubican las fortalezas, las cuales constituyen los ingredientes (procesos o mecanismos) psicológicos que definen las virtudes y, a partir de ellas, se pueden reconocer las virtudes que posee una persona. Finalmente, el nivel más bajo de abstracción está integrado por los temas situacionales que son los hábitos específicos que conducen a una persona a manifestar una determinada fortaleza en una situación específica (Peterson \& Seligman, 2004). 
Tabla 1.

Clasificación de las 6 virtudes y 24 fortalezas (Peterson \& Seligman, 2004).

\begin{tabular}{|c|c|c|}
\hline Virtud & Fortaleza & Definición \\
\hline Coraje & $\begin{array}{l}\text { Valentía } \\
\text { Persistencia } \\
\text { Integridad } \\
\text { Vitalidad }\end{array}$ & $\begin{array}{l}\text { Hacer lo correcto aun corriendo riesgos } \\
\text { Finalizar la tarea a pesar de los obstáculos } \\
\text { Hacer lo que se predica que es correcto } \\
\text { Sentirse vivo y capaz }\end{array}$ \\
\hline Justicia & $\begin{array}{l}\text { Ciudadanía } \\
\text { Liderazgo }\end{array}$ & $\begin{array}{l}\text { Comprometerse con el grupo de pertenencia } \\
\text { Guiar al grupo en armonía hacia buenos resultados } \\
\text { Hacer juicios sociales equitativos y objetivos }\end{array}$ \\
\hline & Imparcialidad & \\
\hline Humanidad & $\begin{array}{l}\text { Amor } \\
\text { Bondad } \\
\text { Inteligencia Social }\end{array}$ & $\begin{array}{l}\text { Considerar muy valioso estar cerca de los afectos } \\
\text { Ayudar a los demás sin esperar nada a cambio } \\
\text { Saber lo que los demás desean y buscan }\end{array}$ \\
\hline Sabiduría & $\begin{array}{l}\text { Perspectiva } \\
\text { Apertura Mental } \\
\text { Amor por el Saber } \\
\text { Curiosidad } \\
\text { Creatividad }\end{array}$ & $\begin{array}{l}\text { Juicio elevado y profundo sobre la vida } \\
\text { Buscar visiones alternativas a las propias } \\
\text { Disfrutar de buscar muchos y mejores conocimientos } \\
\text { Deseo de vivir por experimentar y conocer cosas } \\
\text { nuevas } \\
\text { Poseer ideas originales y aplicarlas }\end{array}$ \\
\hline Templanza & $\begin{array}{l}\text { Clemencia } \\
\text { Humildad } \\
\text { Prudencia } \\
\text { Autorregulación }\end{array}$ & $\begin{array}{l}\text { Comportarse de forma benévola hacia una persona } \\
\text { que no lo merece } \\
\text { No ostentar con los propios logros dejando que } \\
\text { hablen por sí solos } \\
\text { Considerar los pros y contras al contemplar una } \\
\text { decisión } \\
\text { No dejarse llevar por los impulsos y controlar las } \\
\text { respuestas a los mismos }\end{array}$ \\
\hline $\begin{array}{l}\text { Trascen- } \\
\text { dencia }\end{array}$ & $\begin{array}{l}\text { Apreciación } \\
\text { Gratitud } \\
\text { Esperanza } \\
\text { Humor } \\
\text { Espiritualidad }\end{array}$ & $\begin{array}{l}\text { Valorar las cosas lindas de la vida } \\
\text { Sentir y expresar las gracias } \\
\text { Saber que todo estará bien } \\
\text { Tener una mirada de la vida alegre } \\
\text { La vida tiene un sentido superior }\end{array}$ \\
\hline
\end{tabular}

El establecimiento de esta clasificación permitió investigar la existencia de asociaciones entre las fortalezas de carácter y otros constructos psicológicos.

Por ejemplo, Park, Peterson y Seligman (2004) hallaron que las fortalezas de carácter están asociadas con la satisfacción subjetiva con la vida en todas las edades. Si bien Park (2004) afirma que las fortalezas que más contribuyen con la satisfacción vital van cambiando en función del momento evolutivo, estudios en diferentes partes del mundo y dirigidos a distintas franjas etarias parecen indicar que son cinco las fortalezas de carácter que muestran una relación sólida y consistente 
con la satisfacción de la vida en todas las edades : la esperanza, la vitalidad, la gratitud, la curiosidad y el amor (Brdar \& Kashdan, 2010; Brdar, Anić \& Rijavec, 2011; Buschor, Proyer \& Ruch, 2013; Cosentino 2010; Gillman et al., 2011; Giménez, 2010; Littman-Ovadia \& Lavy, 2012; Park \& Peterson, 2006, 2009; Park, Peterson \& Seligman, 2004; Ruch et al., 2010; Proyer, Gander, Wyss \& Ruch, 2011; Ruch et al., 2007; Ruch, Weber, Park \& Peterson, 2014; Shimai et al., 2006).

Asimismo, se encontraron relaciones substanciales entre la clasificación Values in Action con el modelo de personalidad de los Cinco Grandes Factores. Respecto de esto, Park y Peterson (2006a) encontraron que la esperanza, la vitalidad y la autorregulación correlacionaban de manera negativa con Neuroticismo; el humor, el liderazgo, la apertura a la experiencia, la creatividad, el deseo de aprender y la curiosidad correlacionaban con Extraversión; la perseverancia, la prudencia, la autenticidad, la gratitud y la justicia aparecían asociadas con la Responsabilidad, y la generosidad, el amor, la inteligencia social, la ciudadanía y la perspectiva correlacionaban con Amabilidad.

En cuanto a la deseabilidad social, entendida como una predisposición a responder de un modo culturalmente apropiado y aceptable con el objetivo de obtener la aceptación o aprobación de los demás, Peterson y Seligman (2004) afirman que las fortalezas de carácter no están contaminadas por una respuesta de deseabilidad social debido a que son en sí mismas socialmente deseables. Del mismo modo, estos autores no encontraron correlaciones significativas entre las fortalezas de carácter y el índice de deseabilidad social de Marlowe Crowne, a excepción de prudencia $(\mathrm{r}=.44)$ y espiritualidad $(\mathrm{r}=.30)$, en población adulta. Pese a esto, estudios realizados en adolescentes españoles mostraron que todas las fortalezas, a excepción del humor, correlacionaron de forma positiva con la deseabilidad social (Giménez, 2010). Otro importante aporte puede considerarse el de otra investigación realizada en población adulta argentina donde todas las fortalezas del carácter se asociaron de forma positiva y estadísticamente significativa con la deseabilidad social (Cosentino, 2010). Siguiendo con la misma línea de resultados, se pueden mencionar los estudios llevados a cabo en adultos por Macdonald (2008) y en adolescentes por Osin (2009), en los cuales se halló que más de la mitad de las fortalezas correlacionaron con las puntuaciones de una escala que evaluaba deseabilidad social. Finalmente, Ruch, et al. (2010) también encontraron que más de un tercio de las fortalezas se asociaron significativamente con la deseabilidad social.

Con relación a las fortalezas de carácter y variables demográficas, diversos estudios han podido constatar que las mujeres puntúan más alto que los varones en las fortalezas pertenecientes a la virtud humanidad (Brdar et al., 2011; Giménez, 2010; Linley et al., 2007; Ovejero \& Cardenal, 2011; Peterson \& Park, 2003; Peterson \& Seligman, 2004; Ruch et al., 2014; Shimai et al., 2006). También, 
se encontró que a mayor edad hay una mayor presencia de fortalezas tales como la curiosidad, el amor por el conocimiento, la imparcialidad, la clemencia y la autorregulación (Linley et al., 2007). Asimismo, puntos fuertes de carácter tales como la esperanza, la ciudadanía y la vitalidad son más comunes en jóvenes, mientras que la apreciación de la belleza, el liderazgo y la perspectiva aparecen más comúnmente en los adultos (Park \& Peterson, 2003).

La mayoría de las investigaciones sobre fortalezas de carácter están dirigidas a adolescentes y adultos. Sin embargo, la infancia es considerada uno de las etapas propicias para el desarrollo de las mismas (Richards \& Huppert, 2011).

Park (2004) menciona que es muy importante conocer las fortalezas mayormente presentes en el carácter de los niños, ya que esto puede proveer información muy importante a la hora de prevenir diferentes problemas, tales como la agresividad, la adicción al consumo de sustancias y los trastornos depresivos. También, conocer las fortalezas en los niños puede favorecer el éxito escolar y el desarrollo de la autoestima.

A modo de ejemplo, este autor considera que conocer las fortalezas que más se asocian con el bienestar de los niños proporciona pistas muy importantes a la hora de desarrollar programas de intervención basados en las fortalezas del carácter en las escuelas. Este autor propone que aquellas fortalezas más relacionadas con la satisfacción con la vida como la gratitud, el amor, la vitalidad y la esperanza deberían ser las primeras en incluirse en los programas diseñados para el desarrollo positivo de los jóvenes.

En este sentido, la presente investigación tiene como objetivo principal verificar la existencia de asociaciones entre las fortalezas del carácter, el bienestar psicológico, la personalidad y la deseabilidad social en niños de 10 a 12 años de la Ciudad de Buenos Aires.

\section{Método}

\section{Tipo de estudio y diseño}

El presente es un estudio descriptivo-comparativo, con diseño no experimental transversal.

\section{Participantes y procedimiento}

Participaron de este estudio una muestra de conveniencia compuesta por 518 niños que tenían entre 10 y 12 años y que asistían a escuelas públicas y privadas de la Ciudad de Buenos Aires (Argentina). 254 varones (49.1\%) y 263 mujeres (50.9\%). 159 alumnos tenían 10 años (30.8\%), 145 alumnos tenían 11 años (28\%) y 213 alumnos tenían 12 años (41.2\%). La muestra se hallaba balanceada por sexo. El $98 \%$ de los 
padres trabajaban y en su mayoría eran profesionales (47\%), pequeños comerciantes (21\%) o empleados (17\%) y el $70 \%$ de los padres tenía el secundario completo. Se trató de una muestra que, en su mayoría, pertenecía a la clase socioeconómica media. Se solicitó la colaboración voluntaria, anónima y no remunerada de los entrevistados.

Los instrumentos se administraron en forma grupal en las escuelas con un máximo de 30 personas en cada grupo. La participación de los niños estuvo sujeta a su consentimiento y a la previa autorización de sus padres y la institución académica. Tanto los niños como los padres y las autoridades de la institución escolar fueron informados del objetivo de la investigación, garantizando absoluta confidencialidad de los datos obtenidos.

El total de la actividad tuvo una duración de 45 minutos. El análisis de los datos se efectuó con el procesador SPSS versión 19.0.

\section{Instrumentos de recolección de datos}

Encuesta sociodemográfica: Esta encuesta relevó las variables: sexo, edad y nivel académico y ocupacional de los padres de los participantes.

Inventario de Virtudes y Fortalezas Para Niños (IVyF Niños; Grinhauz \& Castro Solano, 2014): Este instrumento está basado en el Inventario de Virtudes y Fortalezas dirigido a población adulta (IVyF; Cosentino \& Castro Solano, 2008). El IVyF Niños es un inventario de papel y lápiz compuesto por 24 ítems de autopuntuación global directa con un formato de respuesta tipo likert de cinco categorías, que van desde el muy parecido a mí a nada parecido a mí. Cada uno de los reactivos evalúa una de las 24 fortalezas correspondientes a la clasificación VIA de Peterson y Seligman (2004). La escala posee adecuadas propiedades psicométricas con un alfa de Cronbach total de .81 (Grinhauz \& Castro Solano, 2014). A continuación se expone un reactivo del IVyF Niños que corresponde a la fortaleza persistencia: "Cuando estudio, no me distraigo ni dejo mis tareas sin terminar. Aunque sea muy dificil lo que estoy haciendo y aunque ya no tenga más ganas de hacerlo, no abandono la tarea y continúo hasta el final".

Encuesta Para Evaluar el Bienestar Psicológico. Esta encuesta está compuesta por seis ítems. Los primeros cinco reactivos se corresponden con los cinco elementos del modelo PERMA de acceso al bienestar (Seligman, 2011). Estos primeros cinco elementos cuentan con un formato de respuesta tipo likert que va desde soy muy parecido hasta soy muy diferente. En el sexto ítem se les pregunta a los participantes ¿Cuán contento/a o satisfecho/a estás con tu vida? Este sexto reactivo cuenta con un formato de respuesta tipo likert de cinco puntos que va desde no estoy contento hasta estoy muy contento o satisfecho con mi vida.

Escala de Deseabilidad Social Infantil (EDESI; Lemos, 2005). Ésta permite evaluar la deseabilidad social en niños de 10 a 12 años. Fue diseñada con ocho 
ítems que consisten en afirmaciones, en las cuales el niño debe responder de forma dicotómica (por sí o por no). Estos ocho reactivos se reparten en dos factores. En el Factor 1 se saturan los cuatro ítems relativos a los aspectos positivos o esperables desde el punto de vista de la deseabilidad social, por ejemplo: "Siempre escucho con atención cuando alguien me habla". En el Factor 2, por su parte, pesaron los cuatro ítems que reflejan los aspectos negativos o socialmente menos esperables, por ejemplo: "Alguna vez me hice como el que entendía algo, cuando en realidad no entendía nada". Se trata de una escala que presenta buenas propiedades psicométricas con un alfa de Cronbach de 70 (Lemos, 2005).

Cuestionario Argentino de Personalidad Infantil (CAPI; Lemos, 2006). Este instrumento consiste en una adaptación del Cuestionario NEOPI-R (Costa \& McCrae, 1980; McCrae \& Costa, 1991; McCrae et al. 2004; McCrae et al., 2005; Costa et al., 2008) que operacionaliza los cinco grandes factores de la personalidad. El CAPI está dirigido a niños de 9 a 12 años, quienes deben responder a los reactivos con sí, no o algunas veces. El análisis factorial exploratorio arrojó 5 factores que explicaron el $54.88 \%$ de la varianza: Neuroticismo (alfa $=.79$ ), Extraversión (alfa $=.80)$, Apertura a la Experiencia (alfa $=.56$ ), Conciencia (alfa $=.71)$ y Amabilidad $($ alfa $=.77)$. A su vez, estos 5 factores contienen 14 facetas de la personalidad (Lemos, 2004): El factor Escrupulosidad contiene las facetas de orden, organización y responsabilidad. El factor Neuroticismo está integrado por las facetas de autocrítica, vulnerabilidad, ansiedad, y en sentido inverso la competencia. El factor Extraversión está integrado por una faceta que atañe a las cuestiones relacionadas con el gregarismo y las emociones positivas. Otra de las facetas que integra este factor es la confianza. En cuanto al factor Amabilidad, la autora decidió nominarlo Mesura vs. Desmesura y contiene la faceta adaptabilidad en contraposición a las facetas de actividad y búsqueda de excitación. Por último, el factor Apertura contiene las facetas de innovación y acción.

\section{Análisis de datos}

Siguiendo el teorema central del límite, dado que los grupos involucrados en las comparaciones de medias superaron los 30 sujetos, se realizaron pruebas paramétricas (Rosner, 2010). Para analizar diferencias entre más de dos medias en grupos independientes se realizaron análisis multivariantes de la varianza (MANOVA). Para determinar en cuáles variables se producían esas diferencias se realizaron posteriormente pruebas univariadas (Peña, 2002). Con el fin de indagar la asociación entre dos variables cuantitativas se llevaron a cabo correlaciones $r$ de Pearson (Pardo \& San Martín, 1999). Al analizar la asociación entre las fortalezas del carácter y la satisfacción con la vida, se decidió llevar a cabo correlaciones parciales controlando por sexo y edad, debido a que, de acuerdo con estudios previos, la 
satisfacción vital podría verse influida por estas variables (Campbell, Converse \& Rodgers, 1976; Clemente, 1996; Fernandez-Ballesteros, 1997; García de León, García de Cortaza \& Ortega, 1996; Hong \& Giannakopoulus, 1994; Klemmack \& Roff, 1984; Lewinson, Redner \& Seeley, 1991; Morgan, Dalloso, Arie, Byrne, Jones \& Waite, 1987; Morganti, Nehrke, Hulicka \& Cataldo, 1988; Quiroga \& Sánchez, 1995; Ryff \& Keyes, 1995; Shmotkin, 1990; Veroff, Douvan \& Kulka, 1981).

\section{Resultados}

\section{Descriptivos}

A continuación, en la tabla 2 se presentan las medias y desviaciones típicas obtenidas en cada fortaleza. En general, se observa que los sujetos presentan puntuaciones medias/altas en casi todas las fortalezas estudiadas (todas están por encima del valor medio de la escala, $>3$, a excepción de amor por el saber). En cuanto a las puntuaciones más altas, se obtienen en las fortalezas gratitud, bondad, apreciación de la belleza y la excelencia, vitalidad y humor (en ese orden). Las medias más bajas pertenecen a amor por el saber, clemencia y autorregulación. 


\section{Tabla 2.}

Descriptivos de las fortalezas de carácter obtenidos mediante el IVyF Niños

\begin{tabular}{lll}
\hline Fortalezas & Media & DE \\
Gratitud & 4.64 & .61 \\
Bondad & 4.55 & .73 \\
Apreciación & 4.36 & .78 \\
Vitalidad & 4.27 & .92 \\
Humor & 4.25 & 1.02 \\
Amor & 4.21 & 1.07 \\
Ciudadanía & 4.19 & .87 \\
Integridad & 4.18 & .95 \\
Liderazgo & 4.11 & .99 \\
Espiritualidad & 4.10 & 1.30 \\
Curiosidad & 4.01 & 1.02 \\
Inteligencia social & 3.99 & 1.21 \\
Imparcialidad & 3.97 & 1.02 \\
Creatividad & 3.90 & 1.16 \\
Apertura mental & 3.88 & 1.09 \\
Prudencia & 3.88 & 1.11 \\
Esperanza & 3.86 & 1.15 \\
Valentía & 3.84 & 1.17 \\
Humildad & 3.79 & 1.24 \\
Persistencia & 3.67 & 1.23 \\
Perspectiva & 3.51 & 1.16 \\
Autorregulación & 3.39 & 1.29 \\
Clemencia & 3.21 & 1.26 \\
Amor por el saber & 2.81 & 1.36 \\
\hline Nota.Apreciacón & . & .29 \\
\hline
\end{tabular}

Nota. Apreciación = apreciación de la belleza; Apertura = apertura mental.

Relaciones entre las fortalezas del carácter y los cinco elementos de acceso al bienestar de la teoría PERMA

Para verificar la existencia de asociaciones entre las fortalezas del carácter y los cinco elementos de la teoría PERMA, se procedió a la realización de correlaciones bivariadas mediante $r$ de Pearson entre las puntuaciones arrojadas por cada una de las cinco viñetas que forman parte de la Encuesta de Bienestar Psicológico y las puntuaciones arrojadas por el IVyF Niños (ver tabla 3).

Con relación al primer elemento de la teoría PERMA, es decir, las emociones 
positivas, puede decirse que se ha asociado de forma positiva y estadísticamente significativa con todas las fortalezas del carácter exceptuando amor por el saber y creatividad. Las asociaciones más elevadas se registraron en las fortalezas vitalidad, apertura mental y curiosidad. Esto significa que el primer elemento de la teoría PERMA - la experimentación de emociones positivas- se encuentra asociado a niños que gozan de buen ánimo, de entusiasmo y energía; a niños reflexivos, con pensamiento crítico, que gustan de explorar y experimentar sin reservas lo novedoso.

El segundo elemento de la teoría PERMA, es decir, el compromiso, se ha asociado de manera positiva y estadísticamente significativa con 20 de las 24 fortalezas del carácter de la clasificación VIA. Las correlaciones más elevadas se registraron en las fortalezas autorregulación, persistencia y prudencia. Esto quiere decir que el compromiso se encuentra asociado a niños capaces de regular sus deseos, necesidades o impulsos siempre que sea necesario, capaces de mantener el curso de la acción a pesar de los obstáculos, y capaces de mantener la cautela y la discreción siempre que la situación lo amerite.

El tercer elemento de la teoría PERMA, el significado vital, se ha asociado de manera positiva y estadísticamente significativa con todas las fortalezas del carácter, hallándose las mayores correlaciones con las fortalezas bondad y vitalidad. Esto significa que el tercer elemento de acceso al bienestar se asocia a niños amables, que gozan de ayudar y cuidar al prójimo, entusiastas y llenos de vigor.

El cuarto elemento, las relaciones sociales positivas, se relacionó de forma positiva y estadísticamente significativa con 20 fortalezas del carácter. Las correlaciones más altas se encontraron con las fortalezas inteligencia social, vitalidad y humor. Esto significa que el cuarto elemento de la teoría PERMA se encuentra asociado a niños expertos en percibir emociones y agudos en el manejo de las relaciones interpersonales; niños que contagian vigor y entusiasmo y que tienen la facilidad para divertirse y disfrutar, la capacidad para enfrentar la adversidad sin perder la alegría y la habilidad para reír y hacer reír a los demás.

Finalmente, el quinto elemento, la autopercepción de logros, se relacionó con 21 fortalezas del carácter. Las mayores correlaciones se encontraron con las fortalezas prudencia, vitalidad y curiosidad. Esto quiere decir que el quinto elemento de acceso al bienestar se encuentra asociado a niños cuidadosos de realizar acciones de las que más tarde se podrían arrepentir, que viven con entusiasmo y energía, con espíritu de aventura y activos a la hora de emprender nuevos aprendizajes. 
Tabla 3.

Correlaciones entre las fortalezas y los cinco elementos de la teoría PERMA.

\begin{tabular}{|c|c|c|c|c|c|}
\hline Fortalezas & $\begin{array}{l}\text { Emocio- } \\
\text { nes positi- } \\
\text { vas }\end{array}$ & $\begin{array}{l}\text { Compro- } \\
\text { miso }\end{array}$ & $\begin{array}{l}\text { Significa- } \\
\text { do vital }\end{array}$ & $\begin{array}{l}\text { Relaciones } \\
\text { sociales } \\
\text { positivas }\end{array}$ & $\begin{array}{l}\text { Autorreali- } \\
\text { zación }\end{array}$ \\
\hline Vitalidad & $.27^{\star \star}$ & $.19^{\star \star}$ & $.32^{\star \star}$ & $.32^{\star \star}$ & $.29^{\star \star}$ \\
\hline Bondad & $.20^{\star \star}$ & $.10^{\star}$ & $.40^{\star \star}$ & $.28^{\star \star}$ & $.25^{\star \star}$ \\
\hline Curiosidad & $.24^{\star \star}$ & $.23^{\star \star}$ & $.28^{\star *}$ & $.20^{\star \star}$ & $.28^{\star \star}$ \\
\hline Prudencia & $.19^{\star \star}$ & $.33^{\star \star}$ & $.28^{\star \star}$ & .08 & $.29^{\star \star}$ \\
\hline Apertura mental & $.25^{\star \star}$ & $.27^{\star \star}$ & $.24^{\star \star}$ & $.20^{\star \star}$ & $.19^{\star \star}$ \\
\hline Liderazgo & $.20^{\star \star}$ & $.16^{\star \star}$ & $.17^{\star \star}$ & $.27^{\star \star}$ & $.22^{\star \star}$ \\
\hline Autorregulación & $.13^{\star \star}$ & $.39^{\star \star}$ & $.17^{\star \star}$ & $.10^{\star}$ & $.17^{\star \star}$ \\
\hline Inteligencia social & $.21^{\star \star}$ & .08 & $.18^{\star \star}$ & $.38^{\star \star}$ & $.16^{\star \star}$ \\
\hline Gratitud & $.23^{\star \star}$ & .09 & $.20^{\star \star}$ & $.28^{\star \star}$ & $.16^{\star \star}$ \\
\hline Esperanza & $.19^{\star \star}$ & $.21^{\star \star}$ & $.17^{\star \star}$ & $.16^{\star \star}$ & $.21^{* *}$ \\
\hline Integridad & $.13^{\star \star}$ & $.18^{\star \star}$ & $.28^{\star \star}$ & $.19^{\star \star}$ & $.15^{\star \star}$ \\
\hline Imparcialidad & $.14^{\star \star}$ & $.19^{\star \star}$ & $.27^{\star \star}$ & $.14^{\star \star}$ & $.15^{\star \star}$ \\
\hline Humildad & $.15^{\star \star}$ & $.16^{\star \star}$ & $.20^{\star \star}$ & $.19^{\star \star}$ & $.16^{\star \star}$ \\
\hline Apreciación & $.13^{\star *}$ & $.15^{\star \star}$ & $.22^{* *}$ & $.12^{\star \star}$ & $.23^{\star \star}$ \\
\hline Ciudadanía & $.15^{\star \star}$ & $.16^{\star \star}$ & $.20^{\star \star}$ & $.19^{\star \star}$ & $.16^{\star \star}$ \\
\hline Persistencia & $.11^{\star \star}$ & $.37^{\star \star}$ & $.10^{*}$ & .08 & $.15^{\star \star}$ \\
\hline Amor & $.14^{\star \star}$ & $.09^{*}$ & $.25^{\star \star}$ & $.16^{\star \star}$ & $.16^{\star \star}$ \\
\hline Clemencia & $.13^{\star \star}$ & $.21^{\star \star}$ & $.24^{\star \star}$ & .04 & $.12^{\star \star}$ \\
\hline Perspectiva & $.11^{\star \star}$ & $.12^{\star \star}$ & $.23^{* *}$ & $.21^{\star \star}$ & $.09^{\star \star}$ \\
\hline Humor & $.13^{\star \star}$ & .03 & $.19^{\star \star}$ & $.30^{\star \star}$ & .06 \\
\hline Amor por el saber & .08 & $.20^{\star \star}$ & $.19^{\star \star}$ & .02 & $.18^{\star \star}$ \\
\hline Valentía & $.10^{*}$ & $.11^{\star \star}$ & $.23^{* *}$ & $.15^{\star \star}$ & .07 \\
\hline Espiritualidad & $.13^{\star \star}$ & $.10^{\star}$ & $.12^{\star \star}$ & $.10^{\star}$ & $.13^{\star \star}$ \\
\hline Creatividad & .04 & .03 & $.23^{\star \star}$ & .07 & $.09^{\star}$ \\
\hline
\end{tabular}

Nota. Se destacan en negrita las correlaciones con tamaños del efecto superiores a mediano $(r=.30$; Cohen, 1992). Apreciación = apreciación de la belleza; Apertura = apertura mental. ${ }^{*} \mathrm{p}<.05$, bilateral. ${ }^{* *} \mathrm{p}<.01$ bilateral. 


\section{Relaciones entre las fortalezas del carácter y la Satisfacción con la Vida}

Para corroborar la existencia de asociaciones existentes entre las fortalezas del carácter y la satisfacción con la vida, se establecieron correlaciones bivariadas mediante $r$ de Pearson entre las puntuaciones arrojadas por la pregunta sobre satisfacción con la vida (¿Cuán satisfecho/a o contento/a te encuentras con tu vida?) y las arrojadas por el IVyF Niños. Posteriormente, se establecieron coeficientes de correlación parcial entre las puntuaciones de la pregunta sobre satisfacción con la vida y el IVyF Niños, controlando por sexo y edad (ver tabla 4).

En los resultados, se encontraron correlaciones estadísticamente significativas y directas entre la satisfacción con la vida y todas las fortalezas de carácter exceptuando creatividad, valentía y perspectiva. En la mayoría de los casos, estas relaciones se mantuvieron significativas al controlar con análisis de covarianza (ANCOVA) las variables sexo y edad, exceptuando las fortalezas apertura, imparcialidad y amor por el saber.

Por otra parte, vale destacar que no se encontraron correlaciones con un tamaño del efecto de moderado a amplio. Las correlaciones más altas fueron entre satisfacción con la vida y las fortalezas vitalidad, esperanza, apreciación de la belleza, curiosidad y prudencia. Esto quiere decir que los niños que señalaron estar más satisfechos con su vida también se autopercibieron más abiertos a explorar y descubrir nuevos temas de interés, con energía para emprender aventuras, cautos a la hora de tomar decisiones o de aconsejar a alguien, con la cualidad de poder percibir la belleza de las cosas simples de la vida y con expectativas positivas respecto del futuro. 
Tabla 4.

Correlaciones entre las fortalezas y la satisfacción con la vida.

\begin{tabular}{|c|c|c|}
\hline Fortalezas & Correlaciones simples & $\begin{array}{l}\text { Correlaciones parciales (contro- } \\
\text { lando por sexo y edad) }\end{array}$ \\
\hline Apreciación & $.23^{\star \star}$ & $.22^{\star \star}$ \\
\hline Imparcialidad & $.11^{\star}$ & .09 \\
\hline Persistencia & $.14^{\star \star}$ & $.13^{\star \star}$ \\
\hline Creatividad & -.02 & -.04 \\
\hline Amor & $.18^{\star \star}$ & $.15^{\star \star}$ \\
\hline Autorregulación & $.18^{\star \star}$ & $.16^{\star \star}$ \\
\hline Gratitud & $.15^{\star \star}$ & $.14^{\star \star}$ \\
\hline Liderazgo & $.15^{\star \star}$ & $.14^{\star \star}$ \\
\hline Apertura mental & $.10^{*}$ & .08 \\
\hline Inteligencia social & $.16^{\star \star}$ & $.16^{\star \star}$ \\
\hline Clemencia & $.15^{\star \star}$ & $.12^{\star \star}$ \\
\hline Espiritualidad & $.18^{\star \star}$ & $.16^{\star \star}$ \\
\hline Ciudadanía & $.13^{\star}$ & $.11^{*}$ \\
\hline Valentía & -.06 & -.06 \\
\hline Curiosidad & $.21^{\star \star}$ & $.19^{\star \star}$ \\
\hline Bondad & $.16^{\star \star}$ & $.13^{\star \star}$ \\
\hline Esperanza & $.23^{\star *}$ & $.22^{\star \star}$ \\
\hline Integridad & $.15^{\star \star}$ & $.13^{\star \star}$ \\
\hline Perspectiva & .03 & .01 \\
\hline Prudencia & $.20^{\star \star}$ & $.17^{\star \star}$ \\
\hline Humor & $.14^{\star \star}$ & $.14^{\star \star}$ \\
\hline Humildad & $.15^{\star \star}$ & $.14^{\star \star}$ \\
\hline Amor por el saber & $.09^{*}$ & .07 \\
\hline Vitalidad & $.25^{\star \star}$ & $.24^{\star *}$ \\
\hline
\end{tabular}

Nota. Apreciación = apreciación de la belleza; Apertura = apertura mental.

${ }^{* *} \mathrm{p}<.01$, bilateral, ${ }^{*} \mathrm{p}<.05$, bilateral

\section{Relaciones entre las fortalezas del carácter y la personalidad}

Para corroborar si se encuentran asociaciones entre las fortalezas de carácter y el modelo de los Cinco Grandes Factores de la Personalidad, se establecieron correlaciones entre las puntuaciones arrojadas por el IVyF Niños y las arrojadas por el CAPI, utilizando $r$ de Pearson (ver tabla 5).

A continuación, se presentan las fortalezas que se asociaron con un tamaño del efecto de moderado a amplio (Cohen, 1992) con la personalidad, ordenadas de forma sucesiva, colocándose primero las fortalezas que obtuvieron un tamaño del efecto más alto. Las fortalezas que se asociaron con el factor Mesura fueron clemencia, prudencia 
y autorregulación. Por otra parte, las fortalezas autorregulación, prudencia, amor por el saber, persistencia, clemencia y curiosidad se asociaron con el factor Escrupulosidad. Todas las correlaciones informadas fueron positivas. Esto quiere decir que los niños con un temperamento más mesurado se reportaron más prudentes, misericordiosos y capaces de regular las propias respuestas frente a los estímulos internos y externos. Asimismo, los niños que se reconocieron más escrupulosos se reportaron con mayor capacidad para regular sus impulsos, más cautos a la hora de tomar decisiones, con una mayor afabilidad por la adquisición de conocimientos nuevos, más persistentes en sus objetivos, misericordiosos y abiertos a explorar y conocer cosas nuevas.

\section{Tabla 5.}

Correlaciones entre las fortalezas del carácter y el modelo de los Cinco Grandes.

\begin{tabular}{|c|c|c|c|c|c|}
\hline Fortalezas & Neuroticismo & Extraversión & Mesura & Escrupulosidad & Apertura \\
\hline Apreciación & -.06 & $.23^{\star \star}$ & .13 & $.20^{\star}$ & $.22^{\star \star}$ \\
\hline Imparcialidad & .01 & .12 & $.25^{\star \star}$ & $.22^{\star \star}$ & .02 \\
\hline Persistencia & -.12 & $.16^{\star}$ & $.25^{\star \star}$ & $.37^{* *}$ & .03 \\
\hline Creatividad & .02 & $.20^{*}$ & -.04 & .15 & .15 \\
\hline Amor & $.22^{\star \star}$ & .10 & $.18^{*}$ & .16 & $.20^{*}$ \\
\hline Autorreg. & -.14 & .03 & $.33^{* *}$ & $.47^{\star \star}$ & .11 \\
\hline Gratitud & -.11 & .07 & .13 & $.30^{* *}$ & .10 \\
\hline Liderazgo & -.11 & .09 & .04 & $.26^{\star *}$ & .12 \\
\hline Apertura & -.01 & .03 & $.23^{* *}$ & $.25^{\star \star}$ & .14 \\
\hline Intelig. Social & -.06 & $.26^{\star \star}$ & .09 & $.21^{\star \star}$ & .10 \\
\hline Clemencia & -.03 & .15 & $.48^{\star \star}$ & $.34^{\star \star}$ & .08 \\
\hline Espiritualidad & .08 & .05 & .15 & .13 & $.17^{\star}$ \\
\hline Ciudadanía & -.11 & $.16^{\star}$ & .08 & $.19^{\star}$ & .10 \\
\hline Valentía & .03 & .11 & .06 & .04 & .15 \\
\hline Curiosidad & -.01 & .08 & .11 & $.32^{* *}$ & $.23^{\star \star}$ \\
\hline Bondad & -.06 & $.26^{\star \star}$ & .10 & $.17^{\star}$ & .14 \\
\hline Esperanza & -.01 & $.22^{\star \star}$ & $.21^{\star \star}$ & .14 & .04 \\
\hline Integridad & .00 & .13 & $.28^{\star \star}$ & $.25^{\star \star}$ & .11 \\
\hline Perspectiva & .01 & $.16^{*}$ & -.04 & .06 & $.21^{\star *}$ \\
\hline Prudencia & .04 & $.19^{*}$ & $.47^{\star \star}$ & $.42^{\star \star}$ & .06 \\
\hline Humor & -.01 & .14 & -.16 & -.01 & .08 \\
\hline Humildad & .00 & .00 & .12 & $.17^{\star}$ & $.19^{\star}$ \\
\hline Amor Saber & .06 & .05 & $.28^{\star *}$ & $.40^{\star \star}$ & .15 \\
\hline Vitalidad & -.13 & $.17^{\star}$ & .15 & $.27^{\star \star}$ & $.16^{\star}$ \\
\hline
\end{tabular}

Nota. Se destacan en negrita las correlaciones con tamaños del efecto superiores a mediano ( $r=.30$; Cohen, 1992). Apreciación = apreciación de la belleza; Autorreg. = Autorregulación; Apertura = apertura mental; Intelig. Social = Inteligencia Social; Amor Saber = Amor por el Saber. ${ }^{*} \mathrm{p}<.05$, bilateral. ${ }^{* *} \mathrm{p}<.01$ bilateral. 
Relaciones entre las fortalezas del carácter y la deseabilidad social

El análisis de datos consistió en la realización de correlaciones bivariadas mediante $\mathrm{r}$ de Pearson entre las puntuaciones arrojadas por la EDESI y las arrojadas por el IVyF Niños (ver tabla 6).

De las 24 fortalezas, 14 se asociaron de forma estadísticamente significativa con la deseabilidad social. Las fortalezas que obtuvieron las más elevadas correlaciones con la deseabilidad social fueron integridad y clemencia -con un tamaño del efecto moderado-.

\section{Tabla 6.}

Correlaciones entre las fortalezas y la deseabilidad social.

\begin{tabular}{|c|c|}
\hline Fortalezas & Deseabilidad Social \\
\hline Integridad & $.38^{\star \star}$ \\
\hline Clemencia & $.37^{\star \star}$ \\
\hline Prudencia & $.29^{* *}$ \\
\hline Autorregulación & $.28^{\star \star}$ \\
\hline Gratitud & $.26^{\star \star}$ \\
\hline Persistencia & $.24^{\star \star}$ \\
\hline Apreciación & $.23^{\star \star}$ \\
\hline Imparcialidad & $.23^{\star \star}$ \\
\hline Apertura mental & $.23^{\star \star}$ \\
\hline Esperanza & $.23^{\star \star}$ \\
\hline Amor por el saber & $.21^{\star \star}$ \\
\hline Vitalidad & $.20^{\star \star}$ \\
\hline Curiosidad & $.18^{\star}$ \\
\hline Creatividad & $.18^{*}$ \\
\hline Espiritualidad & .14 \\
\hline Inteligencia social & .14 \\
\hline Liderazgo & .12 \\
\hline Amor & .10 \\
\hline Bondad & .08 \\
\hline Ciudadanía & .08 \\
\hline Perspectiva & .06 \\
\hline Humildad & .05 \\
\hline Valentía & .03 \\
\hline Humor & .00 \\
\hline
\end{tabular}




\section{Diferencias en las puntuaciones del I.V.YF. Niños de acuerdo al sexo y la edad}

Para contrastar las medias de las puntuaciones del I.V.yF. NIÑOS de acuerdo al sexo y la edad, se realizó un análisis multivariante de la varianza (MANOVA; Peña, 2002) que incluyó al sexo y la edad como factores y las 24 fortalezas de carácter como variables dependientes. Se consideró a la edad como variable discreta, ya que el presente estudio trabaja con tres edades solamente (10, 11 y 12 años). Posteriormente, para corroborar la proporción de la variabilidad total de la variable dependiente que puede ser explicada por el conocimiento de los valores de la variable independiente se calculó el coeficiente Eta Cuadrado ( $\eta 2)$.

En cuanto a las diferencias por sexo, los resultados indican la existencia de diferencias significativas entre los vectores medios de ambos grupos, $T^{2}=0.33$, $F(24,492)=6.93, p<.001$. Para determinar en cuáles fortalezas diferían los varones de las mujeres, se realizaron posteriormente pruebas univariadas (Peña, 2002). Los resultados indicaron que las mujeres puntuaron más alto que los varones en 12 de las 24 fortalezas del carácter. Las mayores diferencias se encontraron en las fortalezas: amor, $M_{\text {mujeres }}=4.55, D E=.74 ; M_{\text {varones }}=3.85, D E=1.23, F(1,515)=60.47 ; p<$ $.001 ; \eta 2=.10 ;$ bondad $M_{\text {mujeres }}=4.75, D E=.50 ; M_{\text {varones }}=4.35, D E=.85, F(1,515)$ $=42.08 ; p<.001 ; \eta 2=.07 ; \mathrm{y}$ perspectiva $M_{\text {mujeres }}=3.75, D E=1.05 ; M_{\text {varones }}=3.27$, $D E=1.21, \mathrm{~F}(1,515)=22.92 ; p<.001 ; \eta 2=.04$.

Con relación a la edad, también se observan diferencias significativas entre los vectores medios de los grupos, $\mathrm{T}^{2}=0.20, F(24,492)=2.11, p<.001$. Al analizar los $\mathrm{F}$ univariados, los niños de 10 años puntuaron más alto que los de 12 años en ocho de las 24 fortalezas. Las mayores diferencias se encontraron en las fortalezas: prudencia, $M_{10 \text { años }}=4.16, D E=.96 ; M_{11 \text { ã̃os }}=3.99, D E=.98, F(2,514)=12.78 ; p$ $<.001 ; \eta 2=.05 ;$ liderazgo, $M_{10 \text { año }} \mathrm{s}=4.28, D E=.84 ; M_{\text {11años }}=4.22, D E=.98, F(2$, $514)=7.68 ; p<.001 ; \eta 2=.03 ;$ y ciudadanía $\mathrm{M}_{10 \text { año }} \mathrm{s}=4.36, D E=.96 ; M_{11 \text { años }}=$ $4.20, D E=.85, F(2,514)=5.84 ; p<.001 ; \eta 2=.02$. Por otra parte, los niños de 11 años puntuaron más alto que los de 12 años en la fortaleza amor, $\mathrm{M}_{11 \text { años }}=4.42$, $D E=.85 ; \mathrm{M}_{12 \text { ã̃os }}=4.06, D E=1.13, F(2,514)=5.08 ; p<.001 ; \eta 2=.02$.

\section{Comentarios}

El objetivo de la presente investigación consistió en verificar la existencia de asociaciones entre las fortalezas del carácter y el bienestar psicológico, la personalidad y la deseabilidad social. Para ello, se trabajó con una muestra de niños de 10 a 12 años que asistían a escuelas públicas y privadas de la Ciudad de Buenos Aires (Argentina).

Con relación a la satisfacción con la vida, se encontraron correlaciones estadísticamente significativas y directas entre este constructo y todas las fortalezas de carácter exceptuando creatividad, valentía y perspectiva. Estos resultados son 
similares a los obtenidos en estudios previos. En este sentido, y de acuerdo con investigaciones previas, la vitalidad, la esperanza y la curiosidad se han encontrado asociadas a la satisfacción vital en todas las edades en diferentes puntos del mundo (Brdar \& Kashdan, 2010; Brdar et al., 2011; Buschor et al., 2013; Cosentino 2010; Gillman et al., 2011; Giménez, 2010; Littman-Ovadia \& Lavy, 2012; Park \& Peterson, 2006, 2009; Park et al., 2004; Park et al., 2010; Proyer et al., 2011; Ruch et al., 2007; Ruch et al., 2014; Shimai et al., 2006).

Respecto de los cinco elementos de la teoría PERMA, se encontraron correlaciones estadísticamente significativas con la mayoría de las fortalezas del carácter tal y como lo advirtió Seligman en el 2011.

Acerca de la deseabilidad social, si bien Peterson y Seligman (2004) no encontraron correlaciones significativas entre las fortalezas de carácter y el índice de deseabilidad social de Marlowe-Crowne (a excepción de prudencia y espiritualidad), en el presente estudio 14 de las 24 fortalezas se asociaron de forma estadísticamente significativa con este constructo. Esto coincide con los resultados encontrados en las investigaciones de Macdonald, Bore y Munro (2008), Osin (2009) y Ruch et al. (2010) en las cuales se halló que más de la mitad de las fortalezas correlacionaban con las puntuaciones de una escala que evaluaba deseabilidad social.

Sobre la asociación existente entre la personalidad y las fortalezas del carácter, debe decirse que los resultados hallados en la presente investigación son bastante similares a los encontrados por Peterson y Seligman (2004). En este sentido, al igual que en los estudios originales, el factor Escrupulosidad (equivalente a Responsabilidad en adultos) registró correlaciones moderadas y significativas con la persistencia y la autorregulación; y el factor Apertura a la Experiencia presentó asociaciones (aunque bajas) con la apreciación de la belleza y la curiosidad.

Por otra parte, se analizaron las diferencias en las fortalezas del carácter de acuerdo con el género y la edad. Los resultados han mostrado que las mujeres puntuaron más alto que los varones en las fortalezas amor y bondad. Estos resultados se encuentran en consonancia con otras investigaciones provenientes de diferentes partes del mundo, las cuales pudieron constatar también que las mujeres suelen obtener puntuaciones más altas que los varones en las fortalezas perteneciente a la virtud humanidad (Brdar et al., 2011; Giménez, 2010; Linley et al., 2007; Ovejero \& Cardenal, 2011; Peterson \& Park, 2003; Peterson \& Seligman, 2004; Ruch et al., 2014; Shimai et al., 2006).

De acuerdo con la edad, estudios previos han mostrado que a mayor edad se espera que exista una mayor presencia de fortalezas (Linley, Joseph, Harrington \& Wood, 2007; Peterson \& Park, 2003; Peterson \& Seligman, 2004). Empero, en otra investigación, Giménez (2010) halló que jóvenes españoles de 10 a 14 años puntuaron más alto en 13 de las 24 fortalezas de la clasificación VIA que los jóvenes 
de mayor edad (15 a 17 años). En la presente investigación, también se encontró que los niños de menor edad (10 años) puntuaron más alto que los de mayor edad (12 años) en ocho de las 24 fortalezas de la clasificación VIA. Las mayores diferencias se encontraron en las fortalezas prudencia, liderazgo y ciudadanía. Esto puede deberse a que, a los 10 años, los niños muestran una mayor preocupación por las normas morales, los juegos grupales comienzan a ser regulados y surge una necesidad imperiosa de respetar las reglas en pos de una convivencia armoniosa con el grupo de pares (Park \& Peterson, 2006; Piaget, 1932).

Con respecto a las limitaciones del presente estudio debe mencionarse la elección de la muestra, que está conformada por instituciones de clase económica media. Sería oportuno en futuras investigaciones contar con una muestra más heterogénea donde participen instituciones educativas de clase alta o clase baja también. Otra de las limitaciones encontradas tiene que ver con el formato de evaluación de los dos instrumentos utilizados, los cuales son autoinformes. Sería acertado para futuros estudios contar con otras fuentes de información.

Pese a las limitaciones mencionadas, se espera que este trabajo haya podido otorgar un poco de claridad en el estudio de los rasgos positivos en niños. De hecho, se lograron conocer las fortalezas del carácter que más se asocian con el bienestar de ellos. Esto proporciona pistas muy importantes a la hora de desarrollar programas de intervención basados en las fortalezas del carácter en las escuelas. Un sistema educativo de calidad debe poner el énfasis no sólo en la transmisión de conocimientos formales, sino también en aspectos tan importantes como la socialización del alumno, el compromiso con la comunidad $\mathrm{y}$ aprendizajes fundamentales que fomenten el desarrollo personal pleno, incluyendo las dimensiones afectivas, sociales, estéticas y morales (Giménez, 2010; Marchesi \& Martín, 1998; Seligman et al., 2009). De acuerdo con Seligman et al. (2009) la escuela debería proveer una educación positiva, cuyo principal objetivo consista en enseñar bienestar a los jóvenes. Así, conocer por ejemplo que la vitalidad, la esperanza y la curiosidad constituyen las fortalezas que más se asocian con la satisfacción con la vida brinda información fundamental para promover una educación positiva a partir del desarrollo de actividades en el aula donde se pongan de manifiesto dichas fortalezas. 


\section{Referencias}

Brdar, I., Anić, P. \& Rijavec, M. (2011). Character Strengths and Well-Being: Are There Gender Differences? En I. Brdar (Ed.), The Human Pursuit of WellBeing: A Cultural Approach (pp. 145-156). New York: Springer.

Brdar, I. \& Kashdan, T. B. (2010). Character strengths and well-being in Croatia: An empirical investigation of structure and correlates. Journal of Research in Personality, 44(1), 151-154.

Buschor, C., Proyer, R. T. \& Ruch, W. (2013). Self-and peer-rated character strengths: How do they relate to satisfaction with life and orientations to happiness? The Journal of Positive Psychology, 8(2), 116-127.

Campbell, A., Converse, P. \& Rodgers, W. (1976). The quality of life: Perceptions, evaluations and satisfactions. New York: Sage.

Castro Solano, A. (2010). Concepciones teóricas acerca de la Psicología Positiva. En A. Castro Solano (Comp.), Fundamentos de Psicología Positiva (pp. 1741). Buenos Aires: Paidós.

Clemente, A. (1996). Psicología del Desarrollo Adulto. Madrid: Narcea.

Cohen, J. (1992). A power primer. Psychological Bulletin, 112(1), 155-159.

Cosentino, A. (2010). Las fortalezas del carácter. En A. Castro Solano (Ed.), Fundamentos de Psicología Positiva (pp. 111-135). Buenos Aires: Paidós.

Costa, P. T., Jr. \& McCrae, R. R. (1980). Influence of extraversion and neuroticism on subjective wellbeing: Happy and unhappy people. Journal of Personality and Social Psychology, 38(4), 668-678.

Costa, P. T., Jr. \& McCrae, R. R. (1991). NEO PI-R. Professional manual. Revised NEO personality inventory (NEO PI-R) and NEO five-factor inventory (NEO-FFI). USA: Psychological Assesment Resources, Inc.

Costa, P. T. \& McCrae, R. R. (1998). Estados de ánimo y personalidad en la etapa adulta. En M. P. Sánchez López \& M. A. Quiroga Estévez (Eds), Perspectivas actuales en la investigación psicológica de las diferencias individuales (pp. 295-310). Madrid: Editorial Centro de Estudios Ramón Areces SA.

Costa, P. T. Jr., McCrae, R. R. \& Martin, T. A. (2008). Incipient adult personality: The NEO-PI-3 in middle-school-aged children. British Journal of 
Developmental Psychology, 26(1), 71-89.

Fernandez-Ballesteros, R. (1997). Calidad de vida en la vejez: Condiciones diferenciales. Intervención psicosocial, 6(1), 21-35.

García de León, M. A., García de Cortaza, M. \& Ortega, F. (1996). Sociología de las mujeres españolas. Madrid: Complutense.

Gillham, J., Adams-Deutsch, Z., Werner, J., Reivich, K., Coulter-Heindl, V., Linkins, M. \& Seligman, M. E. P. (2011). Character strengths predict subjective well-being during adolescence. The Journal of Positive Psychology, 6(1), 31-44.

Giménez, M. (2010). La medida de las fortalezas psicológicas en adolescentes (VIAY): relación con clima familiar, psicopatología y bienestar psicológico. Tesis doctoral no publicada, Facultad de Psicología, Universidad Complutense, Madrid, España.

Grinhauz, A. \& Castro Solano, A. (2014). Validez factorial del Inventario de Virtudes y Fortalezas Para Niños (IVyF NIÑOS). Anuario de Investigaciones, 20(1), 357-361.

Hong, S. M. \& Giannakopoulus E. (1994). The relationship of satisfaction with life to personality characteristics. The Journal of Psychology, 128(5), 547-558.

Klemmack, D. L. \& Roff, L. L. (1984). Fear of personal aging and subjective well-being in later life. Journal of Gerontology, 39, 356-358.

Lambert, N. M., Fincham, F. D., Gwinn, A. M. \& Ajayi, C. A. (2011). Positive relationship science: A new frontier for positive psychology? En K. M. Sheldon, T.B. Kashdan \& M. F. Steger (Eds.), Designing Positive Psychology: taking stock and moving forward (pp. 280-292). Nueva York: Oxford University Press.

Lampropoulos, G. K. (2001). Integrating Psychopathology, Positive Psychology and Psychotherapy. American Psychologist, 56(1), 87-88.

Lemos, V. (2004). Operacionalización del constructo personalidad infantil a partir del enfoque de los Cinco Grandes Factores de Personalidad. Tesis Doctoral para optar por el título de Doctor en Psicología. Universidad Nacional de San Luis.

Lemos, V. (2005). Construcción y validación de una Escala para la Evaluación de la Deseabilidad Social Infantil (EDESI). Interdisciplinaria, 22(1), 79-96. 
Lemos, V. (2006). La evaluación de la personalidad infantil a partir del enfoque de los cinco grandes factores de personalidad (Big Five). Psicodiagnosticar, 16, 97-108.

Lerner, R. M. (1998). Handbook of child psychology: Theoretical models of human development (Vol. 1). New York: Wiley.

Lewinson, P. M., Redner, J. E. \& Seeley, J. R. (1991). The relationship between life satisfaction and psychosocial variables: New perspectives. En F. Strack, M. Argyle, \& N. Schwarz (Eds.), Subjective well-being: An interdisciplinary perspective (pp. 141-169). Oxford, England: Pergamon Press.

Linley, P. A., Maltby, J., Wood, A. M., Joseph, S., Harrington, S., Peterson, C., ... Seligman, M. E. P. (2007). Character strengths in the United Kingdom: The VIA Inventory of Strengths. Personality and individual differences, 43, 341-351.

Littman-Ovadia, H. \& Lavy, S. (2012). Character strengths in Israel: Hebrew adaptation of the VIA Inventory of Strengths. European Journal of Psychological Assessment, 28, 41-50. doi: 10.1027/1015-5759/a000089.

Luthar S. S., \& Cicchetti, D. (2000). The construct of resilience: Implications for interventions and social policies. Development and psychopathology, 12, 857-885.

McCrae, R. R. \& Costa, P. T. (1988). Recalled parent-child relations and adult personality. Journal of Personality, 56, 417-434.

Morganti, J. B., Nehrke, M. F., Hulicka, I. M. \& Cataldo, J. F. (1988). Life-span differences in life satisfaction, selfconcept, and locus of control. International Journal of Aging and Human Development, 26, 45-56.

Ovejero, M. \& Cardenal, V. (2011, julio). Character strengths with gender perspective. A study with a Spanish sample. Trabajo presentado en Second World Congress on Positive Psychology. Philadelphia, Estados Unidos

Park, N. (2004). Character strengths and positive youth development. The Annals of the American Academy of Political and Social Science, 591, 40-54.

Park, N. \& Peterson, C. (2003, marzo). Assessment of character strengths among youth: The Values in Action Inventory of Strengths for Youth. Conferencia presentada en Indicators of Positive Development Conference, Washington, Estados Unidos.

Park, N. \& Peterson, C. (2006a). Character Strengths and happiness among young children: Content analysis of parental descriptions. Journal of Happiness 
Studies, 7, 323-341.

Park, N. \& Peterson, C. (2006b). Moral competence and character strenghts among adolescents: the development and validation of the Values in Action Inventory of Strenghts for Youth. Journal of Adolescence, 29, 891-909.

Park, N., Peterson, C. \& Seligman, M. E. P. (2004). Strengths of character and well-being. Journal of Social \& Clinical Psychology, 23, 603-619.

Peña, D. (2002). Análisis de Datos Multivariantes. Madrid: McGraw-Hill.

Peterson, C. \& Seligman, M. E. P. (2004). Open Mindedness. Judgment, Critical Thinking. En C. Peterson y M.E.P. Seligman (Eds.). Character strengths and virtues: a handbook and classification (pp. 143-159). Washington, DC: American Psychological Association and Oxford University Press.

Quiroga Estévez, M. A. \& Sánchez López, M. P. (1995). Relaciones entre satisfacción familiar y laboral: Variables moduladoras. Anales de Psicología, 11(1), 65-78.

Ruch, W., Huber, A., Beermann, U. \& Proyer, R. T. (2007). Character strengths as predictors of the "good life" in Austria, Germany and Switzerland. En Romanian Academy, "George Barit" Institute of History, Department of Social Research (Ed.), Studies and researches in social sciences (Vol. 16, pp. 123-131). Cluj-Napoca, Romania: Argonaut Press.

Ruch, W., Proyer, R. T., Harzer, C., Park, N., Peterson, C. \& Seligman, M. E. (2010). Values in action inventory of strengths (VIA-IS). Journal of Individual Differences, 31(3), 138-149.

Ruch, W., Weber, M., Park, N. \& Peterson, C. (2014). Character strengths in children and adolescents: Reliability and initial validity of the German Values in Action Inventory of Strengths for Youth (German VIA-Youth). European Journal of Psychological Assessment, 30(1), 57.

Ryff, C. D. \& Keyes, C. (1995). The structure of psychological well-being revisited. Journal of Personality and Social Psychology, 69(4), 719-727.

Seligman, M. E. P. (2002). Authentic happiness: Using the new Positive Psychology to realize your potential for lasting fulfillment. New York: Free Press.

Seligman, M. E. P. (2009). Special Lecture. Documento presentado en Primer Congreso Mundial de Psicología Positiva. International Positive Psychology 
Association, Filadelfia, Pensilvania, Estados Unidos.

Seligman, M. E. P. (2011). Flourish: A visionary new understanding of happiness and well-being. New York: Simon \& Schuster.

Seligman, M. E. P. \& Csikszentmihalyi, M. (2000). Positive Psychology: An introduction. American Psychologist, 55 (1), 5 -14.

Seligman, M. E. P., Parks, A. C. \& Steen, T. (2006). A balanced psychology and a full life. En H. Huppert, B. Keverne \& N. Baylis (Eds.), The science of wellbeing. Oxford: University Press.

Shimai, S., Otake, K., Park, N., Peterson, C. \& Seligman, M. E. P. (2006). Convergence of character strengths in American and Japanese Young Adults. Journal of Happiness Studies, 7, 311-322.

Shmotkin, D. (1990). Subjective well-being as a function of age and gender: A multivariate look for differentiated trends. Social Indicators Research, 23, 201-230.

Steger, M. F. (2009). Meaning in life. En S. J. S. C. R. Lopez (Ed.), Oxford handbook of positive psychology ( $2^{\text {nd }}$ ed., pp. 679-687). New York, NY: Oxford University Press.

Terjesen, M. D. Jacofsky, M. Froh, J. \& DiGiuseppe, R. (2004). Integrating Positive Psychology into schools: Implications for practice. Psychology in the Schools, 41(1), 163-172.

Veroff, J., Douvan, E. \& Kulka, K. (1981). The inner American. New York: Basic Books. 